\title{
Calculation of pair correlations in a high-density electron gas: Constraints for effective interparticle potentials
}

\author{
R. Díez Muiño, ${ }^{1,2}$ I. Nagy, ${ }^{3,2}$ and P. M. Echenique ${ }^{4,2,1}$ \\ ${ }^{1}$ Unidad de Física de Materiales, Centro Mixto CSIC-UPV/EHU, P. Manuel de Lardizabal 3, 20018 San Sebastián, Spain \\ ${ }^{2}$ Donostia International Physics Center, P. Manuel de Lardizabal 4, 20018 San Sebastián, Spain \\ ${ }^{3}$ Department of Theoretical Physics, Institute of Physics, Technical University of Budapest, H-1521 Budapest, Hungary \\ ${ }^{4}$ Departamento de Física de Materiales, Facultad de Químicas, UPV/EHU, Apartado 1072, 20080 San Sebastián, Spain
}

(Received 18 January 2005; revised manuscript received 6 May 2005; published 11 August 2005)

\begin{abstract}
The pair-correlation function $g(r)$ of an interacting, unpolarized electron gas is modelled by using the geminal representation and effective potentials for the two-electron relative motion. We put forward a generalization of the impurity-related Fumi theorem, in order to obtain the scattering-mediated part of the kinetic energy change. Considering the high-density limit, where a perturbatively exact expression for the ground-state energy is available, a rigorous constraint on the effective interactions in the even and odd channels is deduced. The constraint is implemented by using physically motivated one-parametric potentials in these channels.
\end{abstract}

DOI: $10.1103 /$ PhysRevB.72.075117

PACS number(s): 71.10.Ca, 71.45.Gm

\section{INTRODUCTION}

Modern density functional theory (DFT) has had a major impact on electronic structure calculations. ${ }^{1}$ By far, the most popular approximation for this theory is the local density approximation (LDA) for the exchange-correlation energy. The success of the resulting simple computational scheme rests, physically, on a rigorous relation between the exchange-correlation energy and the pair-correlation function. ${ }^{2}$ More precisely, the normalization condition for the exchange-correlation hole continues to hold when the LDA is made for the exact density depletion around an electron. Due to the nature of LDA, the finer microscopical details of the underlying pair-correlation problem of an interacting electron gas are, however, not transparent in this scheme.

A local functional for the exchange-correlation energy, which depends on the local density of occupied states, was introduced recently. ${ }^{3}$ The proposed local parametrization also rests on the homogeneous electron gas model and uses the decomposition of its exchange-correlation hole in scattering states of different relative energies. In this framework, the key quantity is the energy change $e_{x c}\left(k, n_{0}\right)$ of an electron pair with relative momentum $k$ in a system of density $n_{0}$, when the antisymmetrization is imposed and the interaction between particles is switched on. ${ }^{3}$

The idea of pair-approximation to realize the important short-range correlation in the model system directly by using effective (screened) interactions in a two-body Schrödinger equation for the spatial part of two-electron wave functions (geminals) has attracted a considerable interest. ${ }^{4-12}$ Based on the effective potential concept, and focusing on the important pair-correlation function $g(r)$, the description of the standard many-body system can be simplified by transferring a part of complexities from the total wave function to a model Hamiltonian. This intuitive treatment of complexities via effective potentials for particle-interaction is quite similar to the one used in nuclear physics. ${ }^{13}$

A combination of the above ideas is appealing and physically transparent. An approach based on geminals can be useful to discuss the details of the energy change $e_{x c}\left(k, n_{0}\right)$ $\equiv e_{x}\left(k, n_{0}\right)+e_{c}\left(k, n_{0}\right)$. The main goal of the present paper is to describe this quantity in the high density limit. It is this limit in which exact results, in first order of the coupling constant $\alpha \equiv e^{2}$, exist for the on-top value [ $\left.g(r=0)\right]$ of the pair-correlation function and the potential (exchange) energy $\left[\varepsilon_{x}\left(n_{0}\right)\right]$ per particle.

These exact results, should provide natural constraints for describing the $g(r)$ and $e_{c}\left(k, n_{0}\right)$ quantities via geminals and effective interparticle interactions. Similarly as in recent attempts to formulate density matrix functional theory, ${ }^{14,15}$ the prototype many-body system can play here a crucial role in understanding the physical details of dynamical correlation.

The embedded-pair approximation ${ }^{4-12}$ rests, basically, on the scattering aspects of correlated motions. Therefore, similarities and differences in comparison with well-known results obtained for the screening problem of a chargedimpurity are conceptually important.

The rest of the paper is organized as follows. Section II contains a brief summary of the Hartree-Fock approximation in order to show the links to an effective potential approach. In this section, our basic constraint for the kinetic energy change at the high-density limit is formulated using the scattering approach for geminals. The explicit results, obtained by implementing the approach via model potentials, are given there as well. Section III is devoted to a short summary and discussion. We shall use Hartree atomic units in the paper unless otherwise stated.

\section{METHOD AND RESULTS}

The antisymmetry of the state-function under permutation of identical fermions leads to a special correlation between the positions of two such particles whose spins are parallel, even if there is no interaction between the particles. The system is described by a single Slater determinant of momentum eigenstates, i.e., plane waves. In this noninteracting case one has, ${ }^{5}$ in a partial-wave representation for the gemi- 
nals, the following expression for the so-called ideal $\left[g^{0}(r)\right]$ pair distribution function:

$$
g^{0}(r)=\frac{3}{2} \sum_{\substack{\text { odd } l \\ l=1}}^{\infty}(2 l+1)\left\langle j_{l}^{2}(k r)\right\rangle+\frac{1}{2} \sum_{\substack{\text { even } l \\ l=0}}^{\infty}(2 l+1)\left\langle j_{l}^{2}(k r)\right\rangle .
$$

The averages in Eq. (1), denoted by $\langle\cdots\rangle$, are obtained by weighting over the normalized, ideal distribution function $P^{0}(k)$ of the relative momenta ${ }^{13,16}$

$$
P^{0}(k)=24 \frac{k^{2}}{k_{F}^{3}}\left[1-\frac{3}{2} \frac{k}{k_{F}}+\frac{1}{2}\left(\frac{k}{k_{F}}\right)^{3}\right],
$$

where $k_{F}=\left(3 \pi^{2} n_{0}\right)^{1 / 3}$ is the Fermi momentum.

The pair distribution functions are averages for the moving particles. An electron at $r=0$ is not fixed; the reference point moves with the electron. In a geminal-based representation the relative motion is apparent. With $g^{0}(r)$ the electron charge density and the hole-density are $-n_{0} g^{0}(r)$ and $n_{0}\left[g^{0}(r)-1\right]$, respectively. The hole-density is normalized to -1 , and thus one may consider an electron with its hole as a quasiparticle. ${ }^{17}$ We stress the point, that this depletion is prescribed solely by the Pauli's exclusion principle; the HartreeFock hole is not the result of an electrostatic repulsion.

The perturbative potential energy, the exchange energy per particle $\varepsilon_{x}\left(n_{0}\right)$, is given by

$$
\varepsilon_{x}\left(n_{0}\right)=\frac{1}{2} \int_{0}^{\infty} 4 \pi r^{2} \frac{1}{r} n_{0}\left[g^{0}(r)-1\right] d r .
$$

By employing standard results from trigonometry

$$
\begin{aligned}
& \sum_{\substack{\text { odd } l \\
l=1}}^{\infty}(2 l+1) j_{l}^{2}(k r)=\frac{1}{2}\left(1-\frac{\sin 2 k r}{2 k r}\right), \\
& \sum_{\substack{\text { even } l \\
l=0}}^{\infty}(2 l+1) j_{l}^{2}(k r)=\frac{1}{2}\left(1+\frac{\sin 2 k r}{2 k r}\right),
\end{aligned}
$$

we can, after a straightforward manipulation, rewrite Eq. (3) as a function of the relative momentum

$$
\varepsilon_{x}\left(r_{s}\right)=\frac{n_{0}}{2} \int_{0}^{k_{F}} d k P^{0}(k) e_{x}(k) .
$$

In this equation $2 e_{x}(k)=-4 \pi /(2 k)^{2}$, and the Wigner-Seitz parameter $r_{s}$ is defined from $n_{0}=3 /\left(4 \pi r_{s}^{3}\right)$. At the perturbative first-order there is no other contribution to the ideal kinetic energy $\varepsilon_{\text {kin }}^{0}\left(r_{s}\right)=(3 / 10) k_{F}^{2}$.

To obtain the same form for $\varepsilon_{x}\left(r_{s}\right)$ from the more conventional ${ }^{18}$ Wigner-Seitz expression

$$
\varepsilon_{x}\left(r_{s}\right)=-\frac{1}{n_{0}} \int \frac{d^{3} k_{1} d^{3} k_{2}}{(2 \pi)^{6}} n_{k_{1}}^{0} \frac{4 \pi}{\left(\mathbf{k}_{1}-\mathbf{k}_{2}\right)^{2}} n_{k_{2}}^{0},
$$

one should use the $\mathbf{k}=\left(\mathbf{k}_{1}-\mathbf{k}_{2}\right) / 2$ and $\mathbf{s}=\left(\mathbf{k}_{1}+\mathbf{k}_{2}\right) / 2$ variables and perform integrations over these variables under the constraint $^{19}$ of $|\mathbf{s}+\mathbf{k}| \leqslant k_{F}$. In Eq. (7) $n_{k_{i}}^{0}$ refers $(i=1,2)$ to ideal Fermi distribution functions.

The Hartree-Fock wave functions for the homogeneous system are plane waves, thus the above approximation associates interacting particles with a wave function of noninteracting ones and results in the ideal $g^{0}(r)$. Of course, the interaction among particles causes their positions to be correlated, even if there was no symmetrization postulate. Conventionally, the term dynamical correlation is used to name this effect.

Clearly, it is possible to arrange $g(r) \equiv g^{0}(r)+\Delta g(r)$ so that the normalization condition

$$
4 \pi \int_{0}^{\infty} d r r^{2} n_{0} \Delta g(r)=0
$$

is still obeyed, yet the potential energy from Eq. (3) is lower (i.e., larger negative value) than the one found in the HartreeFock approximation. This arrangement will usually cost some kinetic energy change $\left(\varepsilon_{\text {kin }}\right)$, so that one cannot just adjust $\Delta g(r)$ to maximize the potential energy gain $\left(\varepsilon_{\text {pot }}\right)$ alone. Furthermore, these changes are at least second order in the coupling constant $\alpha$ and, in addition, they should satisfy the virial theorem..$^{20,21}$

The pair approximation via effective potentials rests ${ }^{5}$ on the following mathematical observation. The $j_{l}(k r)$ partial waves, which are used to the expansion for geminals in Eq. (1), are scatteringlike (free) solutions of the simple radial Schrödinger equation,

$$
\left(T+\frac{l(l+1)}{2 \mu r^{2}}\right) j_{l}(k r)=\frac{k^{2}}{2 \mu} j_{l}(k r) .
$$

In this equation, $T=[-1 /(2 \mu r)]\left[\left(d^{2} / d r^{2}\right) r\right]$, and the reduced mass is $\mu=1 / 2$.

The intuitive treatment, i.e., the direct approach for an interacting homogeneous system, ${ }^{4-12}$ consists of replacing the noninteracting functions $j_{l}(k r)$ by interaction-based functions $R_{l}(k, r)$ in Eq. (1) and using the $P^{0}(k)$ distribution of Eq. (2). The symmetry-preserving $R_{l}(k, r)$ functions are scattering solutions of the

$$
\left(T+V^{ \pm}(r)+\frac{l(l+1)}{2 \mu r^{2}}\right) R_{l}^{ \pm}(k, r)=\frac{k^{2}}{2 \mu} R_{l}^{ \pm}(k, r),
$$

Schrödinger equation. Here $V^{ \pm}(r)=(\alpha / r) f^{ \pm}(r)$ are screened potentials in even $(+)$ and odd $(-)$ channels. ${ }^{8}$ This choice for the effective potentials is motivated, mainly, by the apparent role of parity in Eq. (1).

By using for $R_{0}^{+}(k, r=0)$ the Lippmann-Schwinger integral equation $^{22}$ and taking the perturbative substitution $\left[j_{0}\left(k r^{\prime}\right)\right.$ $\left.\rightarrow R_{0}^{+}\left(k, r^{\prime}\right)\right]$ in its kernel, one gets

$$
R_{0}^{+}(k, 0)=1-\frac{\mu}{k} \int_{0}^{\infty} d r^{\prime} V^{+}\left(r^{\prime}\right) \sin \left(2 k r^{\prime}\right) .
$$

Based on the rigorous ${ }^{23,24}$ high-density expression for the on-top pair-function $2 g\left(r=0, r_{s}\right)=1-\beta r_{s}$ with $\beta=0.732$, it is easy to show via Eq. (11) that this form is guaranteed by using a one-parametric screened potential with screening pa- 
rameter $\propto k_{F}$. A bare Coulomb potential gives, from the perturbative Lippmann-Schwinger equation, the $2 g\left(r=0, r_{s}\right)=1$ $-6 \pi /\left(5 k_{F}\right)$ expression. This is clearly an overestimation for $\beta$.

The rigorous result was obtained by many-body perturbation theory (up to second order in the coupling, ${ }^{23}$ for the energy) via the static structure function, and by a special double-perturbation approach ${ }^{24}$ for $\Delta g(r)$. This method results in an everywhere nonpositive $\Delta g(r)$ thus the normalization condition for the pair correlation function is not satisfied. The effect leads to overcorrelation. ${ }^{24}$ In the present work, we shall use the exact on-top value for $g\left(0, r_{s}\right)$ as a constraint on $V^{+}(r)$.

Now, let us turn to the exciting problem of characterizing the $k$ dependence of the energy change $e_{c}\left(k, n_{0}\right)$ to the key quantity of a local functional ${ }^{3}$ based on $e_{x c}\left(k, n_{0}\right) \equiv e_{x}(k)$ $+e_{c}\left(k, n_{0}\right)$. This is the input to the equivalent of Eq. (6) for $\varepsilon_{x c}\left(r_{s}\right)$ to describe a ground-state characteristic of a homogeneous, interacting system.

Motivated by the above discussion on the effect of a rearrangement via $\Delta g(r)$, we shall apply the following decomposition: $e_{c}\left(k, n_{0}\right) \equiv e_{\text {kin }}\left(k, n_{0}\right)+e_{\text {pot }}\left(k, n_{0}\right)$. The term describing the potential-energy change is related (in the investigated high-density limit) to an $e^{2}$-order change $\left[\Delta g(r) \propto e^{2}\right]$ in the pair function and to the switching-on of the true Coulomb interaction. This term is, already, at least second order in the coupling constant.

The only remaining term which still may scale linearly with the coupling $\alpha \equiv e^{2}$ in the effective pair approximation for the high density electron gas with certain screened interparticle interactions, is $e_{\text {kin }}\left(k, n_{0}\right)$. Obviously, the correct second-order scaling ${ }^{20,21}$ of the kinetic energy change can prescribe a nontrivial constraint on the geminal-based approach for this system.

In the applied scattering description for $\Delta g(r)$ and $\varepsilon_{\text {kin }}\left(n_{0}\right)$, first we outline how one can rewrite the important normalization condition, Eq. (8), in terms of eigenphase shifts $\delta_{l}^{ \pm}(k)$ of even and odd channels. The standard expression $^{8,25}$ on the volume integral

$$
\int d^{3} r\left[\left|R_{l}^{ \pm}(k, r)\right|^{2}-\left|j_{l}(k r)\right|^{2}\right]=\frac{2 \pi}{k^{2}} \frac{d}{d k} \delta_{l}^{ \pm}(k),
$$

provides the desired link. By changing the order of $k$ and $r$ integrations in Eq. (8), after employing Eqs. (1) and (2), one can easily arrive at the following constraint:

$$
\Delta n^{-}+\Delta n^{+}=0
$$

for which the channel terms are as follows:

$$
\frac{\Delta n^{-}}{n_{0}}=\frac{3}{2} \sum_{\substack{\text { odd } l \\ l=1}}^{\infty}(2 l+1)\left\langle\frac{2 \pi}{k^{2}} \frac{d}{d k} \delta_{l}^{-}(k)\right\rangle,
$$

$$
\frac{\Delta n^{+}}{n_{0}}=\frac{1}{2} \sum_{\substack{\text { even } l \\ l=0}}^{\infty}(2 l+1)\left\langle\frac{2 \pi}{k^{2}} \frac{d}{d k} \delta_{l}^{+}(k)\right\rangle .
$$

In these expressions, the averages over $P^{0}(k)$ are denoted, as before, by $\langle\cdots\rangle$. Further simplifications can be made by employing partial-integrations in making the prescribed averages.

For comparison, in the screening problem of a static embedded charge $(Z)$, say antiproton, in a paramagnetic gas, one has the following Friedel sum on the total induced electron charge $(\Delta n)$ :

$$
\Delta n=\sum_{l=0}^{\infty}(2 l+1) \frac{2}{(2 \pi)^{3}} \int d^{3} k n_{k}^{0} \frac{2 \pi}{k^{2}} \frac{d}{d k} \delta_{l}(k),
$$

as a normalization condition, $\Delta n=Z$ at consistency.

The Friedel condition ensures the charge neutrality of the entire (grand-canonical) system in the presence of the charged impurity. It does not say where the excess electrons are located. This comes from self-consistent-field approximations, where the Poisson equation makes the necessary potential-density connection.

Due to the volume-integral in Eq. (12), there is some similarity between the present and the impurity problems. The essential physical difference is related to the fact that the Hartree-Fock hole is already properly normalized in the paircorrelation problem. The interaction results only in a rearrangement via $\Delta g(r)=g(r)-g^{0}(r)$.

For two particles with relative momentum $k$ interacting via a common potential it is well known ${ }^{26,27}$ that the interaction energy, which is the shift of the energy levels of the system produced by the potential, is given by

$$
\Delta E_{k}=-\frac{2 \pi}{\mu k} \sum_{l=0}^{\infty}(2 l+1) \delta_{l}(k) .
$$

In the case of an embedded impurity $(\mu=1)$, an average of $\Delta E_{k}$ over a Fermi distribution function gives the total energy change in the system. ${ }^{28}$ This change is related to the number $(Z)$ of excess electrons and their energetic redistribution in the field of the external charge. ${ }^{18}$ The kineticenergy change in the grand-canonical system is $Z k_{F}^{2} / 2$, due to the excess electrons. Now we discuss how to obtain these obvious physical results via Eq. (16) and an additional term based on phase shifts.

Let us use, for simplicity, Eq. (10) with a common potential and Eq. (9) for the interaction-free case. Multiplying Eqs. (9) and (10) by $j_{l}(k r)$ and $R_{l}(k, r)$, respectively, and substracting the resulting noninteracting forms from the interacting ones, we perform volume integrations on both sides of the obtained result. The kinetic-energy change $e_{\text {kin }}(l, k)$ in the $l$ channel is given by

$$
e_{\mathrm{kin}}(l, k)=\frac{2 \pi}{\mu k} \delta_{l}(k)+\frac{k^{2}}{2 \mu} \frac{2 \pi}{k^{2}} \frac{d}{d k} \delta_{l}(k),
$$

which contains now, as a first term, the corresponding (negative) interaction-energy term from Eq. (16) on the right-hand side. The second term is based on Eq. (12). 
Summing over $l$ and integrating, as in Eq. (15), over a Fermi distribution function (using $\mu=1$ ) one gets

$$
\varepsilon_{\mathrm{kin}}\left(n_{0}\right)=\frac{2}{\pi} \sum_{l=0}^{\infty}(2 l+1) \int_{0}^{k_{F}} d k \frac{d}{d k}\left(\frac{k^{2}}{2} \delta_{l}(k)\right) .
$$

The above physical statement on the $\varepsilon_{\text {kin }}\left(n_{0}\right)=Z k_{F}^{2} / 2$ value in the impurity-related problem is verified, as Eqs. (15) and (18) nicely show.

The modification via Eq. (17) to our geminal-based problem is straightforward. According to the relative-spin structure we have

$$
e_{\text {kin }}\left(k, n_{0}\right)=e_{\text {kin }}^{-}\left(k, n_{0}\right)+e_{\text {kin }}^{+}\left(k, n_{0}\right),
$$

where the channel contributions are

$$
\begin{aligned}
& \frac{k^{2} e_{\mathrm{kin}}^{-}\left(k, n_{0}\right)}{2 \pi}=\frac{3}{2} \sum_{\substack{\text { odd } l \\
l=1}}^{\infty}(2 l+1) \frac{d}{d k}\left(\frac{k^{2}}{2 \mu} \delta_{l}^{-}(k)\right), \\
& \frac{k^{2} e_{\mathrm{kin}}^{+}\left(k, n_{0}\right)}{2 \pi}=\frac{1}{2} \sum_{\substack{\text { even } l \\
l=0}}^{\infty}(2 l+1) \frac{d}{d k}\left(\frac{k^{2}}{2 \mu} \delta_{l}^{+}(k)\right) .
\end{aligned}
$$

Finally, we perform an average over the $P^{0}(k)$ distribution function to obtain a general expression for the important quantity, the kinetic-energy change

$$
\varepsilon_{\text {kin }}\left(r_{s}\right)=\frac{n_{0}}{2} \int_{0}^{k_{F}} d k P^{0}(k) e_{\text {kin }}\left(k, n_{0}\right) .
$$

Thus, our main goal, i.e., to express these changes by scattering characteristics is formulated.

The important high-density limit is treated via the firstorder Born $(B)$ approximation. This corresponds to the use ${ }^{22}$ of the perturbative form for the phase shifts

$$
\delta_{l}^{ \pm}(k)=-(2 \mu k) \int_{0}^{\infty} d r r^{2} V^{ \pm}(r) j_{l}^{2}(k r) .
$$

To arrive at the perturbative, first order in $e^{2}$, equivalents of Eqs. (13), (14), (19), and (20), we shall use the above expression for the channel phase shifts.

The high-density constraint, $\varepsilon_{\text {kin }}^{B}\left(r_{s} \rightarrow 0\right)=0$, obtained in the Born approximation, could help to avoid overcorrelation, ${ }^{24}$ a possible drawback of modelling. ${ }^{15}$ This averaging to zero in first approximation resembles to the treatment used in nuclear physics to eliminate (in first order) tensor forces in the saturation problem. ${ }^{13}$

A simple inspection of Eqs. (14) and (20) together with Eq. (22) and Eqs. (4) and (5) shows, via order changes in integrations and summations, that in the remaining $k$ integration the $F^{ \pm}(0 ; 2 k) \equiv V^{ \pm}(0) \pm V^{ \pm}(2 k)$ forms will appear in our constraints at the investigated perturbative limit. Here $V^{ \pm}(q)$ is the Fourier transform of the effective $V^{ \pm}(r)$. For example, the equivalent of Eq. (20) in the Born limit is

$$
k^{2} e_{\text {kin }}^{-}\left(k, n_{0}\right)=-\frac{3}{8} \frac{d}{d k}\left[k^{3} F^{-}(0 ; 2 k)\right],
$$

$$
k^{2} e_{\text {kin }}^{+}\left(k, n_{0}\right)=-\frac{1}{8} \frac{d}{d k}\left[k^{3} F^{+}(0 ; 2 k)\right] .
$$

For further analysis, one needs a suitable potential. As we mentioned at Eq. (10) a parity-conserving approximation and the electronic cusp suggest a screened, but Coulombic at $r$ $=0$, potential, $V^{ \pm}(r)=(1 / r) f^{ \pm}(r)$. The above-mentioned important role of $V^{ \pm}(q=0)$ also gives an orientation in illustrative modelling via the deduced, normalization and energetic constraints.

Based on these remarks and previous experiences, ${ }^{10}$ we implement the high-density constraints using the following 29 simple potential:

$$
V^{ \pm}(r)=\frac{e^{2}}{r} e^{-\lambda_{ \pm} r} \cos \left(\lambda_{ \pm} r\right) .
$$

The required Fourier transform of this potential is

$$
V^{ \pm}(q)=4 \pi e^{2} \frac{q^{2}}{q^{4}+4 \lambda_{ \pm}^{4}} .
$$

We shall use in our problem the $\lambda_{+}=0.766 k_{F}$ value which gives ${ }^{10}$ via Eq. (11) the constraining, $2 g\left(0, r_{s} \rightarrow 0\right)=1$ $-0.732 r_{s}$, exact ${ }^{23,24}$ asymptotic form of the pair-correlation function at the origin in the high density limit.

Notice, that at $r=0$ only the $l=0$ partial wave gives contribution. With the above scaling for screening, we have computed the $g\left(0, r_{s}\right)$ function for different $r_{s}$ parameters. The obtained results are exhibited in Fig. 1. A calculation based on an approximation in ladder theory, ${ }^{30}$ results of a numerical solution of an effective Euler-Lagrange equation, ${ }^{31}$ and the exact high-density expression are shown in Fig. 1 as well. It is especially difficult to obtain an accurate value of $g\left(0, r_{s}\right)$ using quantum Monte Carlo methods due to the absence of zero-variance property and arduous numerics. ${ }^{32}$ The ladder-based approximation gives the $2 g\left(0, r_{s} \rightarrow 0\right)=1-0.663 r_{s}$ limiting form. The agreement between the different results is quite reasonable.

After the above fixing of $\lambda_{+}$, we have only the $\lambda_{-}$as free parameter but two constraints, Eqs. (13) to the norm and (21) with $\varepsilon_{\text {kin }}^{B}\left(r_{s}\right)=0$. Therefore, if these conditions could give similar values for $\lambda_{-}$an acceptable consistency will be achieved. Remarkably, it turns out that this is indeed the case. From the normalization constraint we get $\lambda_{-}=1.58 \lambda_{+}$ while from the $\varepsilon^{B}\left(r_{s} \rightarrow 0\right)=0$ energetic constraint we obtain a $\lambda_{-}=1.70 \lambda_{+}$value. Notice, that the similar scaling of a screening parameter with $k_{F}$ was found earlier ${ }^{33}$ by investigating the density-density response function as a solution of the corresponding Bethe-Salpeter equation within a HartreeFock-type theory.

In our parity-conserving approximation with a two parametric, i.e., minimal, model for effective interactions we have $\lambda_{-}>\lambda_{+}$. Therefore, in addition to the obvious effects due to odd or even summations in $l$, we expect smaller changes for $\Delta g_{\uparrow \uparrow}(r)$ than for $\Delta g_{\uparrow \downarrow}(r)$ under the actions of $V^{+}(r)$ and $V^{-}(r)$; these changes are determined mainly by the short-range parts of effective interactions. The additive terms to $2 \Delta g(r)$ are given by 


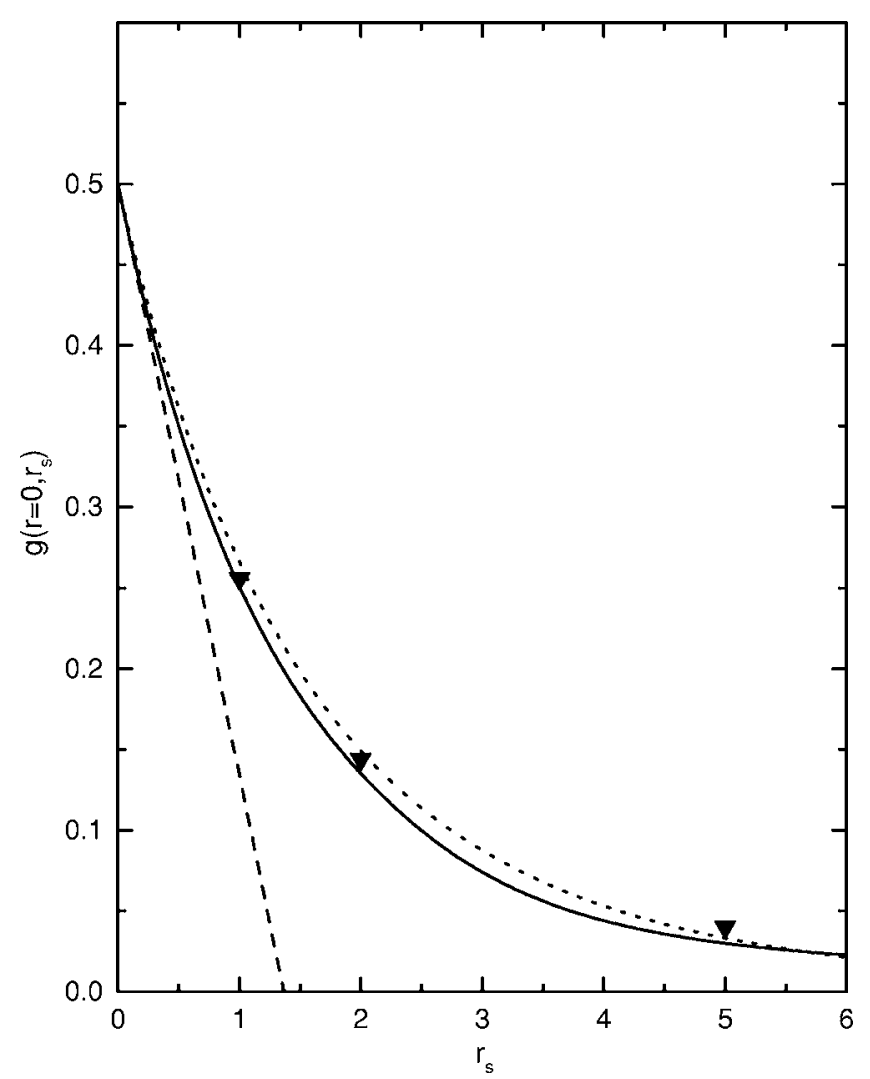

FIG. 1. Pair-correlation function at the origin $g\left(0, r_{s}\right)$ as a function of the electronic density parameter $r_{s}$ (in atomic units). Results obtained in this work are plotted with a solid line. The dotted line refers to the ladder approximation and the dashed line to the exact result in the high density limit. Solid triangles are the results of a numerical solution of an effective Euler-Lagrange equation.

$$
\Delta g_{\uparrow \uparrow}(r)=2\left\langle L^{-}(k, r)\right\rangle
$$

for the parallel-spin component, and

$$
\Delta g_{\uparrow \downarrow}(r)=\left\langle L^{-}(k, r)\right\rangle+\left\langle L^{+}(k, r)\right\rangle
$$

for the antiparallel-spin component of the total change. The $L^{ \pm}(k, r)$ function to $k$ averaging, have the form of

$$
L^{ \pm}(k, r)=\sum_{l}^{ \pm}(2 l+1)\left[\left|R_{l}^{ \pm}(k, r)\right|^{2}-\left|j_{l}(k r)\right|^{2}\right],
$$

where the \pm refer to even $(+)$ and odd $(-)$ in $l$ summations, respectively.

In Fig. 2 we plot our first-order $\Delta g(r) / r_{s}$ function, as a function of the $x=\left(r / r_{s}\right)$ dimensionless variable and taking the Born limit, i.e., $r_{s} \rightarrow 0$. At small $x$ values we have, by construction, the exact limiting value, $-0.732 / 2$, for this function. Note that our method is based on the normalization of $\Delta g(r)$; its components are not constrained separately. These components, from Eqs. (26) and (27), are also exhibited in Fig. 2 via dotted and dashed curves, respectively. The inset shows the above curves around the zero value in an enhanced scale, in order to demonstrate the changes in sign.

As we mentioned earlier, the special double-perturbation approach results in an everywhere nonpositive $\Delta g(r)$ and an

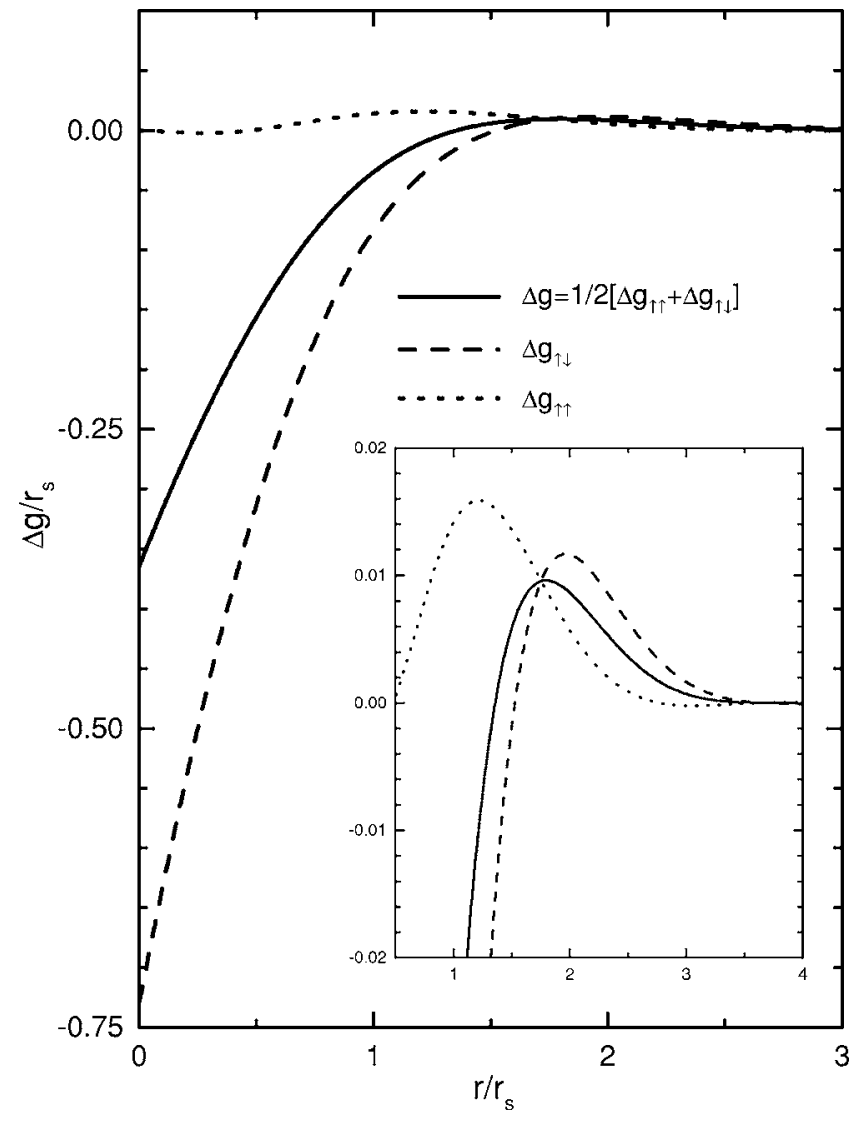

FIG. 2. Difference in the pair-correlation function with respect to the Hartree-Fock case $\Delta g(r)=g(r)-g^{0}(r)$ in the high-density perturbative limit, and as a function of the distance $r$. Both $\Delta g(r)$ and $r$ are divided by $r_{s}$. The solid line is the full $\Delta g(r)$, and the dashed and dotted lines show the antiparallel $\Delta g_{\uparrow \downarrow}(r)$ and parallel $\Delta g_{\uparrow \uparrow}(r)$ contributions to it, respectively. The inset provides a zoom of the $\Delta g(r) \approx 0$ region, to remark the non-negative values of both the parallel and antiparallel contributions to $\Delta g(r)$. All quantities in atomic units.

overcorrelation $^{24}$ because the normalization condition is not satisfied. This approach yields, with common potentials for both the antiparallel and parallel cases, a very similar $\Delta g_{\uparrow \downarrow}(r)$ function for the $x<1$ range; see Fig. 1 of Ref. 24. The shortrange effect of Coulombic repulsion is, in both methods, the same.

On the other hand, the corresponding $\Delta g_{\uparrow \uparrow}(r)$ function is quite different; see Fig. 2 of Ref. 24. It is zero, of course, at $x=0$, but this function has a minimum value of -0.2 at about $x=1$, and is nonpositive everywhere. The observed difference might be related to the not-normalized nature of the approach and, partly, to the fact that in our case we have stronger screening in odd channels than in even channels. Clearly, in the present normalized method the deviation of the exchange-hole from the Hartree-Fock form is very moderate in first order of the true physical coupling.

Now we turn to the presentation of the obtained results for kinetic energy changes which constitute the main motivation to the present work in the high-density, Born limit, in which $\varepsilon^{B}\left(r_{s} \rightarrow 0\right)=0$ at first order of the coupling. The first-order $k_{F}^{2} e_{\text {kin }}^{ \pm}(k) /(2 \pi)$ changes of Eq. (23) and their sum, via Eq. 


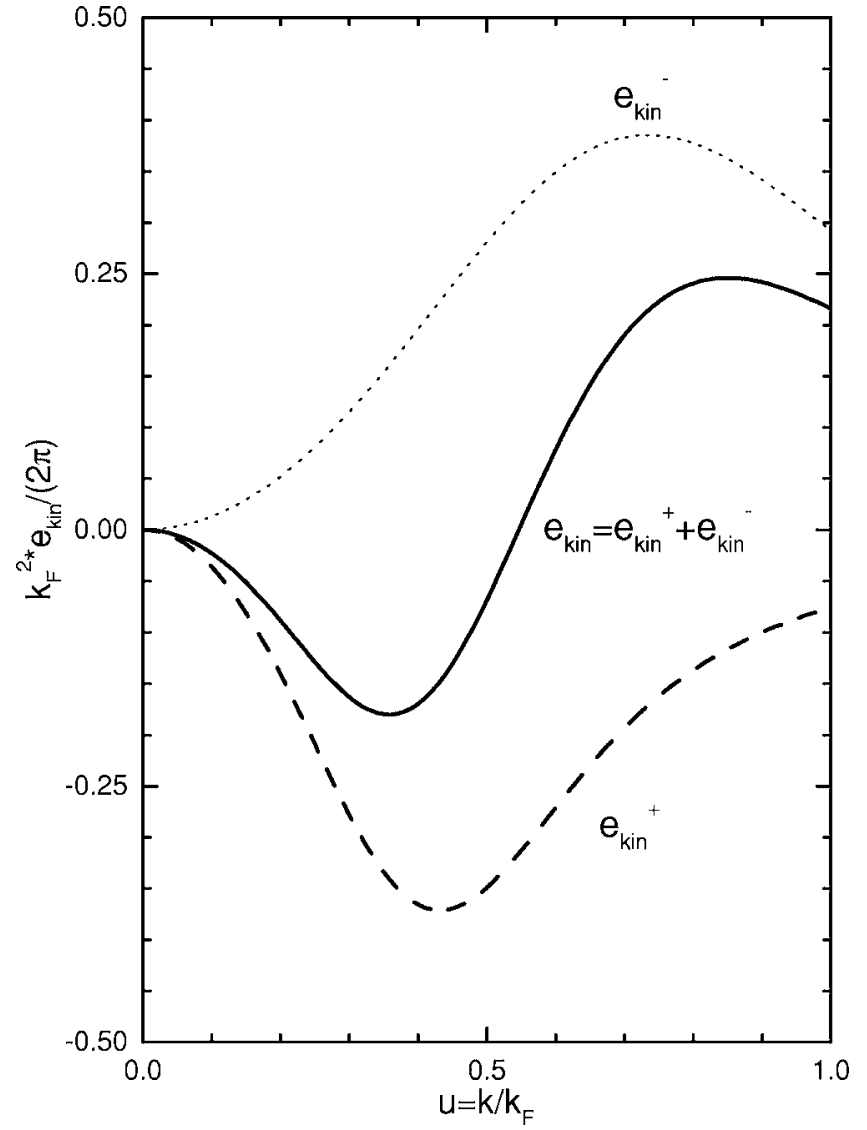

FIG. 3. Kinetic-energy change $e_{\text {kin }}\left(k, n_{0}\right)$ as a function of the dimensionless quantity $k / k_{F}$ (solid line). The odd channel $e_{\text {kin }}^{-}\left(k, n_{0}\right)$ (dotted line) and even channel $e_{\text {kin }}^{+}\left(k, n_{0}\right)$ (dashed line) contributions to $e_{\text {kin }}\left(k, n_{0}\right)$ are shown in the plot as well. The energies $e_{\text {kin }}\left(k, n_{0}\right)$ are multiplied by $k_{F}^{2} /(2 \pi)$. All quantities in atomic units.

(19), are plotted in Fig. 3, as a function of the dimensionless $u=k / k_{F}$ variable.

The formal similarity of Eq. (6) and (21) suggest to use the $k_{F}^{2} e_{x}(k) /(2 \pi)=-1 /(2 u)^{2}$ function as a reference, to understand in a quantitative way the total kinetic energy change, $k_{F}^{2} e_{\text {kin }}(k) /(2 \pi)$. At small relative momenta, this oscillating quantity tends to zero. This limit corresponds to the "comoving" case, kinematically. The net energy change has a maximum value at about the biggest $(u=1)$ relative momenta, the "head-on" case, at which it almost cancels the exchange contribution.

The solid curve crosses the zero value at intermediate, $u$ $\simeq(1 / 2)$, relative momenta. Here appears the nontrivial role of oscillating potentials, via the parity weighting, as the com- parison of the dashed and dotted curves clearly shows. These curves rest on the $\pm V^{ \pm}(2 k)$ forms, because $V^{ \pm}(k=0)=0$ in the present model. Notice that the parity weighting is prescribed solely by the Pauli exclusion principle; we are not considering a symmetry-broken state in our geminal approximation. Finally, in the formal $u \rightarrow \infty$ limit one gets the $\left|e_{x}(k) / e_{\text {kin }}(k)\right|=2$ ratio.

\section{SUMMARY}

In conclusion, motivated by physically transparent ideas, the feasibility of expressing the kinetic energy change of an electron pair with given relative momentum, when the antisymmetrization is imposed and the interaction is switched on, has been investigated. For the high-density electron gas, where some exact results for strongly related characteristics of the system are available, transparent and useful constraints on the effective pair potentials is deduced.

The parity-conserving implementation, via parametric model potentials, gives a consistent physical picture on the rearrangement driven by interparticle interactions. Beyond this perturbative limit, further numerical implementations are needed to establish a practical and well-constrained input to local functionals based on the concept of local density of occupied states.

Beyond the high-density Born approximation, a minimization of the scattering mediated energy change could be a well-motivated constraint for a variational treatment. Such a treatment may have $\mathrm{e}^{34}$ superiority over conventional coupling-constant integration with an approximate potential energy.

Notice, that a recently proposed extension ${ }^{35}$ of the geminal-based idea for inhomogeneous, atomic systems rests also on the minimization of the relative electron-electron kinetic energy. A natural combination of results based on these attempts may provide a deeper understanding of the fine microscopical details of correlated electron motions, which are usually hidden in standard DFT.

\section{ACKNOWLEDGMENTS}

The authors are thankful for useful discussions with J. Soler and J.I. Juaristi. The work of one of the authors (I.N.) has been supported partly by the OTKA (Grants Nos. T046868 and T049571). The authors acknowledge financial support by the Basque Departamento de Educación, Universidades e Investigación, the University of the Basque Country UPV/EHU (Grant No. 9/UPV 00206.215-13639/2001), and the Spanish Ministerio de Educación y Ciencia (Grant No. FIS2004-06490-C03-02).
${ }^{1}$ W. Kohn, Rev. Mod. Phys. 71, 1253 (1998).

${ }^{2}$ O. Gunnarsson and B. I. Lundqvist, Phys. Rev. B 13, 4274 (1976).

${ }^{3}$ J. M. Soler, Phys. Rev. B 69, 195101 (2004).

${ }^{4}$ A. W. Overhauser, Can. J. Phys. 73, 683 (1995).

${ }^{5}$ P. Gori-Giorgi and J. P. Perdew, Phys. Rev. B 64, 155102 (2001).
${ }^{6}$ B. Davoudi, M. Polini, R. Asgari, and M. P. Tosi, Phys. Rev. B 66, 075110 (2002).

${ }^{7}$ I. Nagy, J. I. Juaristi, R. Díez Muiño, and P. M. Echenique, Phys. Rev. B 67, 073102 (2003).

${ }^{8}$ P. Ziesche, Phys. Rev. B 67, 233102 (2003); Phys. Status Solidi B 241, 3544 (2004). 
${ }^{9}$ B. Davoudi, R. Asgari, M. Polini, and M. P. Tosi, Phys. Rev. B 68, 155112 (2003).

${ }^{10}$ I. Nagy, R. Díez Muiño, J. I. Juaristi, and P. M. Echenique, Phys. Rev. B 69, 233105 (2004).

${ }^{11}$ P. Ziesche and F. Tasnádi, Ann. Phys. 13, 232 (2004).

${ }^{12}$ M. Corona, P. Gori-Giorgi, and J. P. Perdew, Phys. Rev. B 69, 045108 (2004).

${ }^{13}$ K. A. Brueckner, Phys. Rev. 96, 508 (1954).

${ }^{14}$ G. Csányi and T. A. Arias, Phys. Rev. B 61, 7348 (2000).

${ }^{15}$ A. Beste and R. J. Bartlett, J. Chem. Phys. 120, 8395 (2004).

${ }^{16}$ N. H. March, W. H. Young, and S. Sampanthar, The Many-Body Problem in Quantum Mechanics (Cambridge University Press, Cambridge, 1967).

${ }^{17}$ P. Fulde, Electron Correlation in Molecules and Solids (SpringerVerlag, Berlin, 1991).

${ }^{18}$ G. D. Mahan, Many-Particle Physics (Plenum, New York, 1981).

${ }^{19}$ L. D. Landau and E. M. Lifshitz, Statistical Physics, Part 1 (Butterworth-Heinemann, Oxford, 1980).

${ }^{20}$ R. A. Ferrell, Phys. Rev. Lett. 1, 443 (1958).

${ }^{21}$ N. H. March, Phys. Rev. 110, 604 (1958).
${ }^{22}$ C. J. Joachain, Quantum Collision Theory (North-Holland, New York, 1975).

${ }^{23}$ J. C. Kimball, Phys. Rev. B 14, 2371 (1976).

${ }^{24}$ V. A. Rassolov, J. A. Pople, and M. A. Ratner, Phys. Rev. B 59, 15625 (1999).

${ }^{25}$ I. Nagy and A. Bergara, Nucl. Instrum. Methods Phys. Res. B 115, 58 (1996).

${ }^{26}$ C. J. Pethick and G. M. Carneiro, Phys. Rev. A 7, 304 (1973).

${ }^{27}$ S. J. J. M. F. Kokkelmans, G. V. Shlyapnikov, and C. Salomon, Phys. Rev. A 69, 031602(R) (2004).

${ }^{28}$ F. G. Fumi, Philos. Mag. 46, 1007 (1955).

${ }^{29}$ I. Nagy and A. Bergara, J. Phys.: Condens. Matter 11, 3943 (1999).

${ }^{30}$ H. Yasuhara, Solid State Commun. 11, 1481 (1972).

${ }^{31}$ A. Kallio and J. Piilo, Phys. Rev. Lett. 77, 4237 (1996).

${ }^{32}$ W. M. C. Foulkes, L. Mitas, R. J. Needs, and G. Rajagopal, Rev. Mod. Phys. 73, 33 (2001).

${ }^{33}$ G. E. Engel, Phys. Rev. Lett. 78, 3515 (1997).

${ }^{34}$ A. W. Overhauser and J. T. Tsai, Phys. Rev. B 13, 607 (1976).

${ }^{35}$ P. Gori-Giorgi and A. Savin, Phys. Rev. A 71, 032513 (2005). 